\title{
Evaluating Users' Emotion in Web-Based Geographic Information System
}

\author{
Leni Novianti ${ }^{1}$, Indra Griha Tofik Isa ${ }^{1,{ }^{*}}$, Indri Ariyanti ${ }^{1}$, Rika Sadariawati ${ }^{1}$, \\ Anitawati Mohd Lokman², Azhar Bin Abd Aziz², Afiza Binti Ismail ${ }^{2}$
}

\author{
${ }^{1}$ Politeknik Negeri Sriwijaya, Palembang, Indonesia \\ ${ }^{2}$ Universiti Teknologi MARA, Shah Alam, Malaysia \\ *Corresponding author. Email: indra_isa_mi@polsri.ac.id
}

\begin{abstract}
GIS is one of the tools ease the user for get information based on map images that have been processed. In addition to technical aspects, GIS needs to involve psychological factors and user emotions so as to provide user comfortable. Kansei Engineering is an engineering method that involves psychological factors and user emotions. This research is an initial analysis to evaluate the emotional aspects of GIS users that have high significance and will then be involved in the Kansei Engineering stage in making recommendations for GIS User Interface. The Initial Study involved 80 Participants with a distribution of 40 female and 40 male, 50 Emotions / Kansei Words, 7 Specimens. The method stages are (1) Preparation of Instruments consisting of specimens, Kansei Word and Evaluation Subjects, (2) Evaluation, (3) Analysis using Cronbach's Alpha, Coefficient Correlation Analysis (CCA) and Factor Analysis (FA). The study result 15 emotions / Kansei Words that have significant based on CCA and FA comparison result for GIS Web UI domain that consist of "Accurate", "Brilliant", "Sharp", "Wonderful", "Dynamic", "Beautiful", "Wide", "Well-Arranged”, "Cool”, “Authentic", "Elegant”, "Formal”, "Masculine”, "Easy-to-Measure” and "Bright”.
\end{abstract}

Keywords: Kansei, Emotion, Web-Based-GIS

\section{INTRODUCTION}

Geographic Information System (GIS) is a tool to represent certain conditions so that the public can know the phenomena that occur, for example the distribution of forest fire data, population demographics, the distribution of Covid-19 data, and so on [1]. Currently, various GIS variations are presented, both in the form of dynamic and static mappings that are match to the needs of the user. Generally, the information presented in a GIS is representative for the user, because it contains data and information related to the presented domain [2]. Mostly, in its development it has not concern to ergonomic aspects and user aspect from psychological and emotional factors.

Kansei Engineering (KE) is one of the methods used in product development by involving psychological factors and user emotions [3]. KE can be implemented in GIS development, especially the User Interface aspect, how to produce a display that meets ergonomic aspects by involving psychological factors and user emotions [4]. The thing that must be considered in the KE stage is the quality of the emotions or $\mathrm{KW}$ involved which have a significant influence value so that they represent psychological or emotional factors from the user [5] [6]

This study a pilot analysis that aims to assess the quality of $\mathrm{KW}$ or emotion factor which has a significant value and see the potential of users and participants who have sensitivity to $\mathrm{KW}$. The $\mathrm{KW}$ involved in the study amounted to $50 \mathrm{KW}$ that were taken from various references, including: Personal Perception, GIS user perception, programmer perception, general user perception. While the participants involved were 80 participants with the criteria of 40 male participants and 40 female participants, and using 7 web-based GIS specimens 


\section{RESEARCH METHOD}

The pilot analysis purposes is finding significant emotion for GIS Web UI Domain and valid kansei evaluation subject. Figure 1 shows the phases of the feelings, emotions and psychology. If it does not meet these elements, then an exclude statement is given, and if it is inaccurate but is a word that describes the user's emotions, then a rephrase statement is given on the.

The initial KW that has been identified then
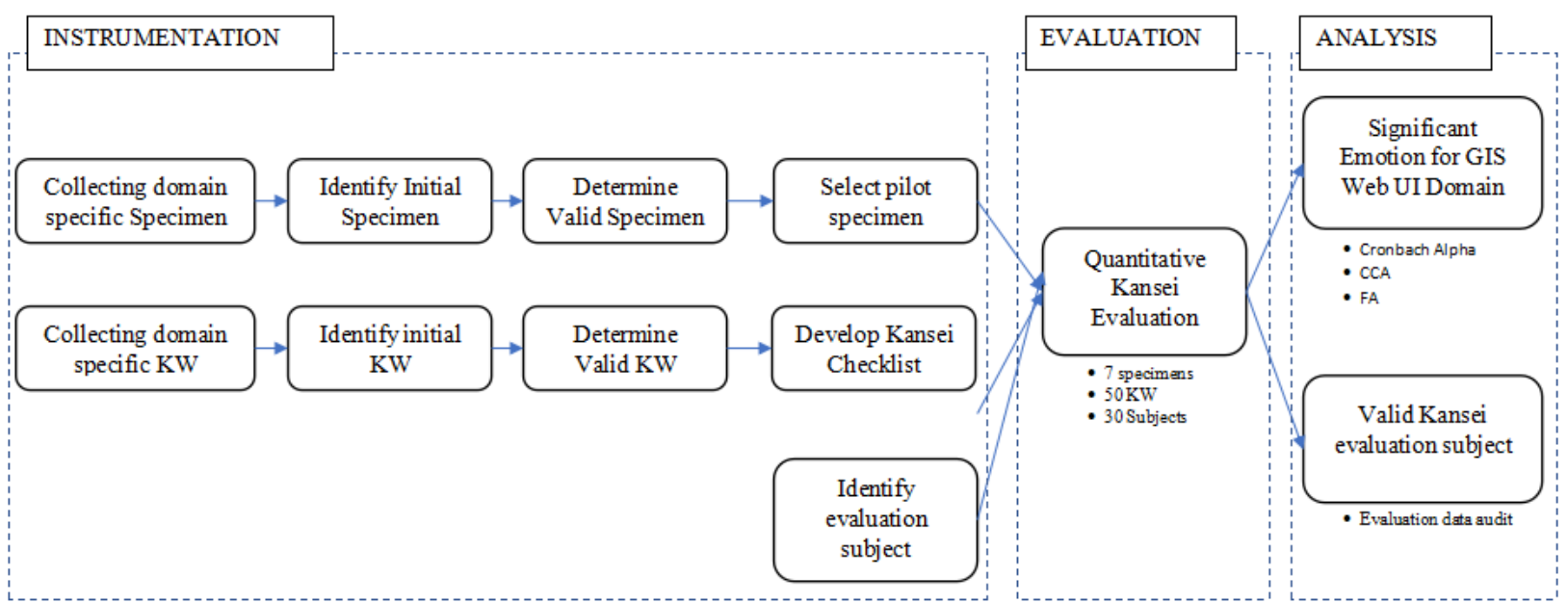

Figure 1. Research Method

study:

In this study there are 3 steps as shown in Figure 1, they are:

\subsection{Prepare the Instrumentation}

There are 3 aspects to focus on in preparing the instrumentation, namely (1) Selecting pilot specimen, (2) Develop Kansei Checklist, (3) Identify Evaluation Subject. In selecting pilot specimens, it is started by collecting domain specific specimens. The collection of specimens was obtained from the GIS website with various variations. The collected specimen data is then identified by involving various aspects related to UI such as background, color characteristic, theme, font characteristic and others. Furthermore, determining the valid specimen where the specimen has different characteristics between specimens. In this pilot analysis there are 7 specimens selected to be involved in Kansei Evaluation.

Meanwhile in developing the Kansei Checklist started with collecting domain specific Kansei Word $(\mathrm{KW})$ which is a representation of the user's emotional factors, starting with the stage of collecting KW taken from several sources, including the perceptions of researchers, GIS users, general users and web designers. $\mathrm{KW}$ retrieval is done by giving all specimens to the user, then the user gives a statement in the form of what feelings feel after seeing the interface of the specimen. Expressions that are shown verbally are then identified to see KW which can provide elements of the user's determined to be involved in pilot analysis. There are 50 $\mathrm{KW}(\mathrm{s})$ that will be included in Kansei Checklist. In Developing Kansei Checklist using Semantic Differential Scale (SD Scale) with 5 scale range. The lowest score is 1 , and the highest score is 5 . For the questionnaire instrument with the lowest score / number 1 , the word "Not" is added, while the highest value / number 5 is added the word "Very".

In the evaluation stage, instruments were distributed to participants. The instrument consisted of 7 specimens and $50 \mathrm{KW}$ and 80 participants. The technique of filling in the instrument is done online via google form. The user is given an access link to the filling instrument, then the user fills in the name and KW questionnaire based on the specimen image contained in the instrument. Users can view specimen images in the google form, also equipped with detailed image links.

\subsection{Analysis}

The results of filling out the questionnaire were then averaged and data analysis was performed to see Significant Emotion for GIS Web UI Domain and Valid Kansei evaluation subject. The analysis was carried out using Cronbach's Alpha, Coefficient Correlation Analysis (CCA) and Factor Analysis (FA). From the results of this analysis, it can be seen which $\mathrm{KW} /$ emotion has a high significance. Meanwhile, to determine the Valid Kansei evaluation subject, it is carried out by evaluating audit data 


\section{RESULT}

\subsection{Instrumentation}

\subsubsection{Specimen}

Specimen determination is done by referencing several web-based mappings. Due to the limited number of websites related to web-based regional asset mapping, specimens refer to websites that are integrated into the Geographic Information System. There are 26 prospective specimens to be sorted where unique specimens are sought in terms of coloring, menus, components, map characteristics and other aspects related to the User Interface. Table 1 shows 26 specimen collection results:

Table 1. Collecting Specimens Result

\begin{tabular}{|c|c|c|c|}
\hline No & Specimen & No & Specimen \\
\hline 1 & $\begin{array}{l}\text { Peta Sebaran Cov-19 } \\
\text { Indonesia }\end{array}$ & 14 & $\begin{array}{|lr|}\text { USGS. National } & \text { Water } \\
\text { Information } & \text { System: } \\
\text { Mapper } & \end{array}$ \\
\hline 2 & $\begin{array}{l}\text { Covid-19 Data } \\
\text { Explorer: Global } \\
\text { Humanitarian } \\
\text { Operations }\end{array}$ & 15 & $\begin{array}{l}\text { Sabah Tourism - Sipadan } \\
\text { Island }\end{array}$ \\
\hline 3 & $\begin{array}{l}\text { WHO Coronavirus } \\
\text { (Covid-19) Dashboard }\end{array}$ & 16 & $\begin{array}{l}\text { US-EPA. National Aquatic } \\
\text { Resource Surveys }\end{array}$ \\
\hline 4 & Open Street Map & 17 & Water Risk Atlas \\
\hline 5 & $\begin{array}{l}\text { Peta Online ATR / BPN } \\
\text { (Badan Pertanahan } \\
\text { Nasional) }\end{array}$ & 18 & Atlas Online \\
\hline 6 & Portal Peta Indonesia & 19 & 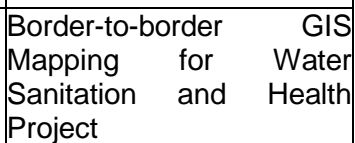 \\
\hline 7 & $\begin{array}{l}\text { Pemetaan Kementerian } \\
\text { ESDM Indonesia }\end{array}$ & 20 & $\begin{array}{l}\text { Florida Department of } \\
\text { Environmental Protection }\end{array}$ \\
\hline 8 & Land Portal & 21 & $\begin{array}{l}\mid \text { TNB's Asset (Power Plant, } \\
\text { Transmission Networks, } \\
\text { Distribution Networks, } \\
\text { Fiber Optics Cable and } \\
\text { Customers' meter) to be } \\
\text { Pinned Down on Digital } \\
\text { Map }\end{array}$ \\
\hline 9 & $\begin{array}{l}\text { Science for a Changing } \\
\text { World }\end{array}$ & 22 & $\begin{array}{ll}\text { Malaysia } & \text { Covid-19 } \\
\text { Dashboard } & \end{array}$ \\
\hline 10 & $\begin{array}{l}\text { Waze - Navigation \& } \\
\text { Life Traffic }\end{array}$ & 23 & \begin{tabular}{|l} 
Peta Hospital Rujukan \\
Covid-19, Pusat Kuarantin \\
dan Kemudahan Awam
\end{tabular} \\
\hline 11 & MAGIC Website (UK) & 24 & $\begin{array}{|lr|}\text { GeoBencana rrabaha } & \text { Pejabat } \\
\text { Setuausaha } & \text { Kerajaan } \\
\text { Negeri Pulau Pinang }\end{array}$ \\
\hline 12 & $\begin{array}{l}\text { Sea Grant University of } \\
\text { Winconsin }\end{array}$ & 25 & $\begin{array}{l}\text { NT Atlas and Spatial Data } \\
\text { Directory }\end{array}$ \\
\hline 13 & $\begin{array}{l}\text { Malaysia Covid-19 } \\
\text { Dashboard }\end{array}$ & 26 & Esri Map Gallery \\
\hline
\end{tabular}

From the 26 collecting specimen, then identified initial based on general User Interface aspect, such as background theme, color theme, font style, font color, the characterisic of top menu, left menu, body, right menu and other. Table 2 shows the result of idetifying initial specimen:

Table 2. Identifying Specimens Result

\begin{tabular}{|c|l|c|}
\hline No & \multicolumn{1}{|c|}{ Specimen } & Result \\
\hline $\mathbf{1}$ & Peta Sebaran Cov-19 Indonesia & OK \\
\hline $\mathbf{2}$ & $\begin{array}{l}\text { Covid-19 Data Explorer: Global } \\
\text { Humanitarian Operations }\end{array}$ & OK \\
\hline $\mathbf{3}$ & WHO Coronavirus (Covid-19) Dashboard & OK \\
\hline $\mathbf{4}$ & Open Street Map & OK \\
\hline $\mathbf{5}$ & $\begin{array}{l}\text { Peta Online ATR / BPN (Badan } \\
\text { Pertanahan Nasional) }\end{array}$ & OK \\
\hline $\mathbf{6}$ & Portal Peta Indonesia & OK \\
\hline $\mathbf{7}$ & Pemetaan Kementerian ESDM Indonesia & OK \\
\hline $\mathbf{8}$ & Land Portal & OK \\
\hline $\mathbf{9}$ & Science for a Changing World & Not OK \\
\hline $\mathbf{1 0}$ & Waze - Navigation \& Life Traffic & Not OK \\
\hline $\mathbf{1 1}$ & MAGIC Website (UK) & OK \\
\hline $\mathbf{1 2}$ & Sea Grant University of Winconsin & Not OK \\
\hline $\mathbf{1 3}$ & Malaysia Covid-19 Dashboard & Not OK \\
\hline $\mathbf{1 4}$ & $\begin{array}{l}\text { USGS. National Water Information } \\
\text { System: Mapper }\end{array}$ & Not OK \\
\hline $\mathbf{1 5}$ & Sabah Tourism - Sipadan Island & OK \\
\hline $\mathbf{1 6}$ & $\begin{array}{l}\text { US-EPA. National Aquatic Resource } \\
\text { Surveys }\end{array}$ & Not OK \\
\hline $\mathbf{1 7}$ & Water Risk Atlas & OK \\
\hline $\mathbf{1 8}$ & Atlas Online & OK \\
\hline $\mathbf{1 9}$ & $\begin{array}{l}\text { Border-to-border GIS Mapping for Water } \\
\text { Sanitation and Health Project }\end{array}$ & Not OK \\
\hline $\mathbf{2 0}$ & $\begin{array}{l}\text { Florida Department of Environmental } \\
\text { Protection }\end{array}$ & OK \\
\hline $\mathbf{2 1}$ & $\begin{array}{l}\text { TNB's Asset (Power Plant, Transmission } \\
\text { Networks, Distribution Networks, Fiber } \\
\text { Optics Cable and Customers' meter) to be } \\
\text { Pinned Down on Digital Map }\end{array}$ & OK \\
\hline $\mathbf{2 2}$ & Malaysia Covid-19 Dashboard & OK \\
\hline $\mathbf{2 3}$ & $\begin{array}{l}\text { Peta Hospital Rujukan Covid-19, Pusat } \\
\text { Kuarantin dan Kemudahan Awam }\end{array}$ & Not OK \\
\hline $\mathbf{2 4}$ & $\begin{array}{l}\text { GeoBencana Pejabat } \\
\text { Kerajaan Negeri Pulau Pinang }\end{array}$ \\
\hline $\mathbf{2 5}$ & NT Atlas and Spatial Data Directory & OK \\
$\mathbf{2 6}$ & Esri Map Gallery & OKaha \\
\hline
\end{tabular}

From the 26 specimens identified, then determined 7 specimen that will be involved in evaluation phase. Table 3 shows the 7 specimens that involved in evaluation:

Table 3. 7 Specimens involved in Evaluation

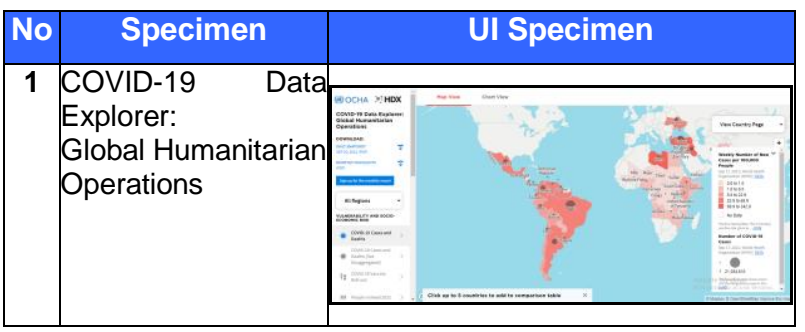




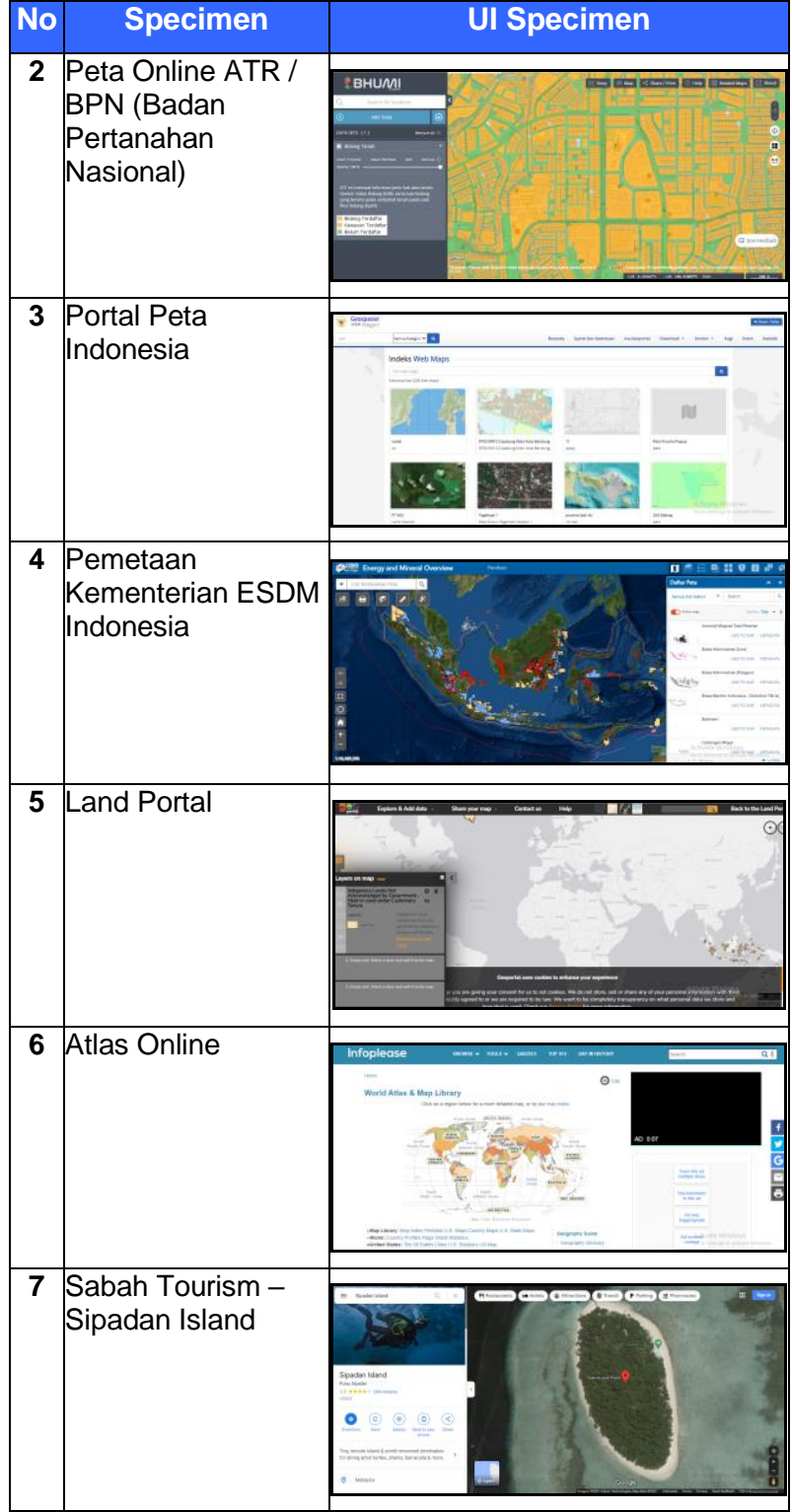

\subsubsection{Kansei Word}

Kansei Word is taken from several sources including the perception of the research team, user perception, and web designer's perception. The technique of collecting Kansei Word is done by giving specimens to several respondents to give their perception of their feelings when they see the specimen. These perceptions become Kansei Word candidates that need to be validated. After validation, 50 Kansei Words are generated which represent the Web-Based GIS specimens shown in table 4
Table 4. Kansei Word Result

\begin{tabular}{|c|l|l|l|}
\hline Kode & Kansei Word & \multicolumn{1}{|c|}{ Kode } & Kansei Word \\
\hline KW1 & Sharp & KW 26 & Useful \\
\hline KW 2 & Formal & KW 27 & Wonderful \\
\hline KW 3 & Simple & KW 28 & Beautiful \\
\hline KW 4 & Informative & KW 29 & Elegant \\
\hline KW 5 & Gloomy & KW 30 & Brilliant \\
\hline KW 6 & Dynamic & KW 31 & Impressive \\
\hline KW 7 & Rigid & KW 32 & Authentic \\
\hline KW 8 & Natural & KW 33 & Easy-to- \\
& & & Measure \\
\hline KW 9 & Calm & KW 34 & Empty \\
\hline KW 10 & Sad & KW 35 & Sophisticated \\
\hline KW 11 & Prestigious & KW 36 & Easy \\
\hline KW 12 & Masculine & KW 37 & Vivid \\
\hline KW 13 & Bright & KW 38 & Colorful \\
\hline KW 14 & Fresh & KW 39 & Complex \\
\hline KW 15 & Catchy & KW 40 & Bored \\
\hline KW 16 & Nautical-Look & KW 41 & Complete \\
\hline KW 17 & Wide & KW 42 & Global \\
\hline KW 18 & Well-Arranged & KW 43 & Melancholic \\
\hline KW 19 & Accurate & KW 44 & Free \\
\hline KW 20 & Cool & KW 45 & Stiff \\
\hline KW 21 & Awesome & KW 46 & Modern \\
\hline KW 22 & Trustworthy & KW 47 & Confusing \\
\hline KW 23 & Cold & KW 48 & Common \\
\hline KW 24 & Cute & KW 50 & Look-Tired \\
\hline KW 25 & Classic & & \\
\hline
\end{tabular}

\subsection{Evaluation}

Evaluation is done by using google form, Kansei Word is translated to google form instrument with adding specimen to make it easy for participant for fulfilling the instrumen. The instrument is defined by 7 part based on 7 instruments. The figure of specimen is added to the instrument. Figure 1 shows the instrument distributed to participant :

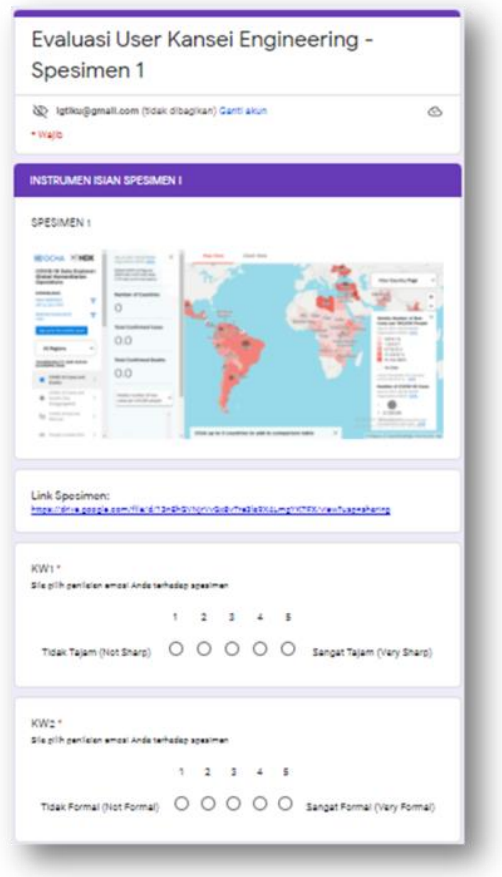

Figure 2. Instrument's Participant 
The data collected using instrument then converted into spreadsheet to make calculation and analysis easier, as shown in table 5:

Table 5. Data Participant Result

\begin{tabular}{|c|c|l|c|c|c|c|c|c|}
\hline No & Participant & Sex & KW1 & KW2 & KW3 & KW4 & $\ldots$ & KW50 \\
\hline 1 & Participant 1 & Female & 4 & 4 & 5 & 5 & & 4 \\
\hline 2 & Participant 2 & Male & 2 & 3 & 1 & 3 & $\ldots$ & 5 \\
\hline 3 & Participant 3 & Female & 3 & 3 & 2 & 2 & $\ldots$ & 4 \\
\hline 4 & Participant 4 & Female & 5 & 2 & 3 & 3 & $\ldots$ & 4 \\
\hline 5 & Participant 5 & Female & 3 & 4 & 1 & 3 & $\cdots$ & 3 \\
\hline 6 & Participant 6 & Female & 5 & 5 & 5 & 5 & $\cdots$ & 3 \\
\hline 7 & Participant 7 & Female & 4 & 4 & 1 & 4 & $\cdots$ & 4 \\
\hline 8 & Participant 8 & Male & 4 & 4 & 2 & 3 & $\ldots$ & 4 \\
\hline$\ldots$ & $\ldots$ & $\ldots$ & $\cdots$ & $\ldots$ & $\cdots$ & $\ldots$ & $\ldots$ & $\ldots$ \\
\hline 80 & Participant 80 & Male & 5 & 4 & 4 & 5 & $\cdots$ & 3 \\
\hline
\end{tabular}

Then the data participant result from 7 instruments are recapitulated. Table 6 shows the recapitulation of participant's evaluation with the total 80 participant consists of 40 female dan 40 male:

Table 6. Recapitulation of Participant's Evaluation

\begin{tabular}{|c|l|l|c|c|c|c|c|c|c|}
\hline No & Description & Sex & KW1 & KW2 & KW3 & KW4 & KW5 & $\ldots$ & KW50 \\
\hline 1 & Participant 1 & Male & 3,29 & 2,71 & 2,43 & 3,86 & 2,71 & $\ldots$ & 3,29 \\
\hline 2 & Participant 2 & Female & 3,71 & 4,43 & 4,29 & 4,43 & 2,71 & $\ldots$ & 1,43 \\
\hline 3 & Participant 3 & Male & 2,43 & 4,14 & 4,29 & 4,57 & 1,71 & $\ldots$ & 1,29 \\
\hline 4 & Participant 4 & Female & 3,86 & 3,86 & 2,86 & 4,29 & 2,43 & $\ldots$ & 2,57 \\
\hline 5 & Participant 5 & Male & 3,29 & 3,00 & 3,29 & 4,14 & 1,00 & $\ldots$ & 1,00 \\
\hline 6 & Participant 6 & Female & 3,57 & 2,86 & 3,29 & 3,43 & 3,00 & $\ldots$ & 2,57 \\
\hline 7 & Participant 7 & Male & 3,14 & 4,14 & 4,14 & 4,43 & 4,29 & $\ldots$ & 3,43 \\
\hline 8 & Participant 8 & Male & 4,29 & 4,43 & 3,57 & 4,86 & 1,57 & $\ldots$ & 2,57 \\
\hline$\ldots$ & $\ldots$ & $\ldots$ & $\ldots$ & $\ldots$ & $\ldots$ & $\ldots$ & $\ldots$ & $\ldots$ & $\ldots$ \\
\hline 80 & Participant 80 & Male & 3,00 & 3,14 & 3,14 & 3,57 & 1,43 & $\ldots$ & 2,00 \\
\hline
\end{tabular}

\subsection{Analysis}

The results of the participant recapitulation were then averaged based on the specimen for analysis as shown in table 7.
Table 7. Average Evaluation Data

\begin{tabular}{|c|c|c|c|c|c|c|c|}
\hline $\begin{array}{c}\text { Specimen } \\
\text { ID }\end{array}$ & $\mathbf{1}$ & $\mathbf{2}$ & $\mathbf{3}$ & $\mathbf{4}$ & $\mathbf{5}$ & $\mathbf{6}$ & $\mathbf{7}$ \\
\hline KW1 & 3,46 & 3,44 & 3,19 & 4,28 & 2,34 & 2,70 & 2,70 \\
\hline KW2 & 3,56 & 3,38 & 3,63 & 4,13 & 3,25 & 3,24 & 3,24 \\
\hline KW3 & 3,03 & 2,48 & 3,38 & 2,81 & 3,46 & 3,13 & 3,13 \\
\hline KW4 & 4,30 & 3,59 & 4,01 & 4,45 & 3,39 & 3,79 & 3,79 \\
\hline KW5 & 2,38 & 2,99 & 2,10 & 2,11 & 3,54 & 2,55 & 2,55 \\
\hline KW6 & 3,51 & 3,18 & 3,45 & 3,93 & 2,88 & 3,05 & 3,05 \\
\hline KW7 & 2,70 & 3,54 & 2,83 & 2,51 & 3,54 & 2,98 & 2,98 \\
\hline KW8 & 3,55 & 2,99 & 3,48 & 4,08 & 2,88 & 3,10 & 3,10 \\
\hline KW9 & 3,55 & 2,70 & 3,54 & 3,71 & 3,15 & 3,06 & 3,06 \\
\hline$\ldots$ & $\ldots$ & $\ldots$ & $\ldots$ & $\ldots$ & $\ldots$ & $\ldots$ & $\ldots$ \\
\hline KW50 & 2,60 & 3,36 & 2,26 & 2,11 & 3,10 & 2,79 & 2,79 \\
\hline
\end{tabular}

\subsubsection{Cronbach's Alpha}

Cronbach's Alpha is involved to measure of internal consistency how closely related a set of items are as a group that range value between 0 to 1 [7]. In this study, the Cronbach's Alpha value generated from 80 respondents with and $50 \mathrm{KW}$ is $\mathbf{0 . 9 3 1}$ as shown in table 8 .

Table 8. Cronbach'a Alpha Statistic

\begin{tabular}{|c|c|}
\hline $\begin{array}{c}\text { Cronbach's } \\
\text { alpha }\end{array}$ & $\begin{array}{c}\text { Standardized } \\
\text { Cronbach's Alpha }\end{array}$ \\
\hline 0,931 & 0,939 \\
\hline
\end{tabular}

Cronbach's Alpha score of 0.931 is included in the high category because it is greater than $>0.60$ so it can be concluded that all instrument items are consistent and reliable [7].

\subsubsection{Coefficient Correlation Analysis (CCA)}

CCA is used to see the correlation of $50 \mathrm{KW}$ which has a high influence value of other KW. CCA uses the XLStat 2021 tool. The CCA results are in table 9 below:

\section{Table 9. Coefficient Correlation Analysis Result}

\begin{tabular}{|c|c|c|c|c|c|c|c|c|}
\hline & KW1 & KW2 & KW3 & KW4 & KW5 & KW6 & $\cdots$ & KW50 \\
\hline KW1 & $\mathbf{1}$ & $\mathbf{0 , 9 0 3}$ & $-0,639$ & $\mathbf{0 , 8 0 0}$ & $-0,625$ & $\mathbf{0 , 9 3 6}$ & $\cdots$ & $-0,541$ \\
\hline KW2 & $\mathbf{0 , 9 0 3}$ & $\mathbf{1}$ & $-0,276$ & $\mathbf{0 , 8 3 1}$ & $-0,643$ & $\mathbf{0 , 9 6 7}$ & $\cdots$ & $-0,770$ \\
\hline KW3 & $-0,639$ & $-0,276$ & $\mathbf{1}$ & $-0,200$ & $\mathbf{0 , 1 1 2}$ & $-0,339$ & $\cdots$ & $-0,231$ \\
\hline KW4 & $\mathbf{0 , 8 0 0}$ & $\mathbf{0 , 8 3 1}$ & $-0,200$ & $\mathbf{1}$ & $-\mathbf{0 , 8 6 4}$ & $\mathbf{0 , 9 2 7}$ & $\boldsymbol{\cdots}$ & $-0,845$ \\
\hline KW5 & $-0,625$ & $-0,643$ & 0,112 & $-\mathbf{- 0 , 8 6 4}$ & $\mathbf{1}$ & $\mathbf{- 0 , 7 6 7}$ & $\boldsymbol{\cdots}$ & 0,852 \\
\hline
\end{tabular}




\begin{tabular}{|c|c|c|c|c|c|c|c|c|}
\hline KW6 & 0,936 & 0,967 & $-0,339$ & 0,927 & $-0,767$ & 1 & $\ldots$ & $-0,789$ \\
\hline KW7 & $-0,592$ & $-0,697$ & $-0,032$ & $-0,942$ & 0,912 & $-0,793$ & $\cdots$ & 0,920 \\
\hline KW8 & 0,856 & 0,954 & $-0,189$ & 0,953 & $-0,794$ & 0,977 & $\cdots$ & $-0,879$ \\
\hline KW9 & 0,515 & 0,773 & 0,309 & 0,826 & $-0,659$ & 0,777 & $\cdots$ & $-0,916$ \\
\hline KW10 & $-0,255$ & $-0,232$ & 0,245 & $-0,127$ & 0,502 & $-0,219$ & $\cdots$ & 0,315 \\
\hline KW11 & 0,829 & 0,908 & $-0,139$ & 0,960 & $-0,804$ & 0,966 & $\cdots$ & $-0,859$ \\
\hline KW12 & 0,870 & 0,956 & $-0,343$ & 0,664 & $-0,396$ & 0,874 & $\cdots$ & $-0,578$ \\
\hline KW13 & 0,756 & 0,606 & $-0,470$ & 0,850 & $-0,913$ & 0,771 & $\cdots$ & $-0,623$ \\
\hline KW14 & 0,882 & 0,899 & $-0,305$ & 0,972 & $-0,886$ & 0,971 & $\ldots$ & $-0,844$ \\
\hline$\ldots$ & $\ldots$ & $\ldots$ & $\ldots$ & $\ldots$ & $\ldots$ & $\ldots$ & $\ldots$ & $\ldots$ \\
\hline KW50 & $-0,818$ & $-0,471$ & $-0,896$ & 0,569 & $-0,615$ & $-0,813$ & $\ldots$ & 1 \\
\hline
\end{tabular}

From the table of CCA results above, it can be seen that there are several KWs that have high influence values. In the CCA analysis this refers to a value greater than 0.9. Some of them are KW21 with the emotion "Awesome" which has a correlation with a value above 0.9 against the other $28 \mathrm{KW}$, namely KW1 or "Sharp", KW4 or "Informative", KW06 or "Dynamic", KW8 or "Natural" and so on. From CCA result, top $25 \mathrm{KW}$ that have correlation score to other $\mathrm{KW}$ more than 0.9 is shown in table 10:

\section{Table 10. Top 25 CCA Result}

\begin{tabular}{|c|l|l|c|l|l|}
\hline No & Kode & \multicolumn{1}{|c|}{ Emotion } & No & Kode & \multicolumn{1}{|c|}{ Emotion } \\
\hline 1 & KW21 & Awesome & 14 & KW22 & Trustworthy \\
\hline 2 & KW30 & Brilliant & 15 & KW4 & Informative \\
\hline 3 & KW31 & Impressive & 16 & KW26 & Useful \\
\hline 4 & KW27 & Wonderful & 17 & KW16 & Nautical-Look \\
\hline 5 & KW20 & Cool & 18 & KW15 & Catchy \\
\hline 6 & KW14 & Fresh & 19 & KW37 & Vivid \\
\hline 7 & KW11 & Prestigious & 20 & KW2 & Formal \\
\hline 8 & KW8 & Natural & 21 & KW19 & Accurate \\
\hline 9 & KW6 & Dynamic & 22 & KW1 & Sharp \\
\hline 10 & KW46 & Modern & 23 & KW18 & Well-Arranged \\
\hline 11 & KW35 & Sophisticated & 24 & KW38 & Colorful \\
\hline 12 & KW27 & Wonderful & 25 & KW23 & Cold \\
\hline 13 & KW28 & Beautiful & & & \\
\hline
\end{tabular}

\subsubsection{Factor Analysis}

Factor analysis was conducted to see the concept of emotion that appear from the participants. From the Factor Analysis, 5 factors (F1 - F5) were produced after varimax rotation, as shown in table 11 :

Table 11. Factor Variability Table

\begin{tabular}{|c|c|c|}
\hline & Variability (\%) & Cumulative \% \\
\hline F1 & 49,902 & 49,902 \\
\hline F2 & 24,777 & 74,679 \\
\hline F3 & 9,243 & 83,923 \\
\hline F4 & 8,998 & 92,921 \\
\hline F5 & 7,079 & 100 \\
\hline
\end{tabular}

In table $11 \mathrm{~F} 1$ produces a factor value of $49.902 \%$ and $\mathrm{F} 2$ is $24.777 \%$. If it is accumulated between F1 and $\mathrm{F} 2$, it results in an accumulation value of $74,679 \%$, as well as furthermore on F3, F4 and F5, the accumulation percentage increases to $100 \%$. However, the factors that have a high value are F1 and F2 because they cumulatively produce more than $70 \%$. Table 12 shows specifically the $\mathrm{KW}$ contained in F1 and F2 sorted in ascending order.

Table 12. Sorted FA Result

\begin{tabular}{|c|c|c|c|c|c|c|}
\hline KW & F1 & KW & F2 & KW & F3 & .. \\
\hline KW34 & $-0,850$ & KW25 & $-0,884$ & KW39 & $-0,964$ & $\ldots$ \\
\hline KW40 & $-0,843$ & KW5 & $-0,780$ & KW45 & $-0,510$ & $\ldots$ \\
\hline$\ldots$ & $\ldots$ & $\ldots$ & $\ldots$ & $\ldots$ & $\ldots$ & $\ldots$ \\
\hline KW46 & 0,819 & KW16 & 0,480 & KW37 & 0,065 & $\ldots$ \\
\hline KW21 & 0,824 & KW21 & 0,499 & KW29 & 0,079 & $\ldots$ \\
\hline KW22 & 0,838 & KW31 & 0,505 & KW8 & 0,105 & $\ldots$ \\
\hline KW8 & 0,844 & KW22 & 0,528 & KW11 & 0,176 & $\ldots$ \\
\hline KW31 & 0,847 & KW26 & 0,564 & KW36 & 0,208 & $\ldots$ \\
\hline KW19 & 0,853 & KW46 & 0,568 & KW10 & 0,222 & $\ldots$ \\
\hline KW30 & 0,854 & KW11 & 0,576 & KW42 & 0,238 & $\ldots$ \\
\hline KW1 & 0,888 & KW14 & 0,597 & KW16 & 0,244 & $\ldots$ \\
\hline KW27 & 0,891 & KW36 & 0,604 & KW33 & 0,250 & $\ldots$ \\
\hline KW6 & 0,891 & KW35 & 0,619 & KW19 & 0,268 & $\ldots$ \\
\hline KW28 & 0,892 & KW15 & 0,636 & KW24 & 0,283 & $\ldots$ \\
\hline KW17 & 0,897 & KW37 & 0,646 & KW48 & 0,304 & $\ldots$ \\
\hline KW18 & 0,902 & KW38 & 0,679 & KW34 & 0,305 & $\ldots$ \\
\hline KW20 & 0,929 & KW4 & 0,693 & KW25 & 0,307 & $\ldots$ \\
\hline KW32 & 0,935 & KW41 & 0,703 & KW44 & 0,365 & $\ldots$ \\
\hline KW29 & 0,962 & KW33 & 0,719 & KW9 & 0,571 & $\ldots$ \\
\hline KW2 & 0,965 & KW24 & 0,763 & KW23 & 0,634 & $\ldots$ \\
\hline KW12 & 0,995 & KW13 & 0,803 & KW3 & 0,923 & $\ldots$ \\
\hline
\end{tabular}

In table 12 , the F1 value taken is more than 0.85 which produces $13 \mathrm{KW}$ that is consist of "Accurate", "Brilliant", "Sharp", "Wonderful", "Dynamic", "Beautiful", "Wide", “ Well-Arranged", "Cool", "Authentic", "Elegant", "Formal", "Masculine"; F2 consists of $4 \mathrm{KW}$ with the emotions "Complete", "Easyto-Measure", "Cute" and "Bright"; F3 consists of KW3 "Simple"; F4 consists of KW10 "Sad" and KW49 "Creepy" and F5 consists of "Global".

Then is comparing the results of the FA with the CCA, for example in F1 there is KW19 with the emotion "Accurate". The results from the CCA show that KW19 has a correlation value with other KWs 
which is greater than the value of 0.7 for 29 correlation, greater than the value of 0.8 for 23 relations and greater than 0.9 for 7 relations. Table 13 is a comparison of the results of FA Factor 1 with CCA:

Table 13. Comparison Factor 1 Result and CCA

\begin{tabular}{|c|c|c|c|c|c|}
\hline \multirow{2}{*}{ No } & \multirow{2}{*}{ KW } & \multirow{2}{*}{$\begin{array}{c}\text { Factor 1 } \\
\text { Result }\end{array}$} & & \multicolumn{3}{|c|}{ CCA Result } \\
\cline { 4 - 6 } & & $\mathrm{n}>\mathbf{0 . 7}$ & $\mathrm{n}>\mathbf{0 . 8}$ & $\mathrm{n}>\mathbf{0 . 9}$ \\
\hline 1 & KW19 & $\mathbf{0 . 8 5 3}$ & 29 & 23 & 7 \\
\hline 2 & KW30 & $\mathbf{0 . 8 5 4}$ & 32 & 28 & 20 \\
\hline 3 & KW1 & $\mathbf{0 . 8 8 8}$ & 29 & 22 & 11 \\
\hline 4 & KW27 & $\mathbf{0 . 8 9 1}$ & 31 & 27 & 29 \\
\hline 5 & KW6 & $\mathbf{0 . 8 9 1}$ & 32 & 27 & 19 \\
\hline 6 & KW28 & $\mathbf{0 . 8 9 2}$ & 32 & 26 & 19 \\
\hline 7 & KW17 & $\mathbf{0 . 8 9 7}$ & 24 & 11 & 4 \\
\hline 8 & KW18 & $\mathbf{0 . 9 0 2}$ & 25 & 20 & 5 \\
\hline 9 & KW20 & $\mathbf{0 . 9 2 9}$ & 28 & 27 & 16 \\
\hline 10 & KW32 & $\mathbf{0 . 9 3 5}$ & 25 & 19 & 8 \\
\hline 11 & KW29 & $\mathbf{0 . 9 6 2}$ & 29 & 26 & 16 \\
\hline 12 & KW2 & $\mathbf{0 . 9 6 5}$ & 29 & 24 & 16 \\
\hline 13 & KW12 & $\mathbf{0 . 9 9 5}$ & 24 & 17 & 7 \\
\hline
\end{tabular}

From the table comparing factor 1 with CCA, it can be seen that the number of correlations that have a value of more than 0.7 are mostly above 24 correlations between KWs. For the number of KW with "n > 0.7", the highest number is KW30 emotion "Brilliant", KW6 emotion "Dynamic" and KW28 "Beautiful". Meanwhile for "n > 0.9" is KW20 with "cool" emotion. The emotions contained in table 13 are classified as having a high significance value because based on the CCA analysis they have a high correlation value, also based on the FA analysis they have a high factor value above 0.8 . The results of the comparative analysis between factor 2 and CCA can be seen in table 14 below.

Table 14. Comparison Factor 2 Result and CCA

\begin{tabular}{|c|c|c|c|c|c|}
\hline \multirow{2}{*}{ No } & \multirow{2}{*}{ KW } & \multirow{2}{*}{$\begin{array}{c}\text { Factor 2 } \\
\text { Result }\end{array}$} & \multicolumn{4}{|c|}{ CCA Result } \\
\cline { 4 - 6 } & & & $\mathbf{n}>\mathbf{0 . 7}$ & $\mathbf{n}>\mathbf{0 . 8}$ & $\mathrm{n}>\mathbf{0 . 9}$ \\
\hline 1 & KW41 & $\mathbf{0 . 7 0 3}$ & 18 & 6 & 1 \\
\hline 2 & KW33 & $\mathbf{0 . 7 1 9}$ & 20 & 10 & 2 \\
\hline 3 & KW24 & $\mathbf{0 . 7 6 3}$ & 16 & 6 & 1 \\
\hline 4 & KW13 & $\mathbf{0 . 8 0 3}$ & 22 & 9 & 2 \\
\hline
\end{tabular}

In table 14 above, KW33 and KW13 have more than 20 correlations for " $\mathrm{n}>0.7$ " with other KWs in the CCA, and this is still a $\mathrm{KW}$ with a high significance value. Furthermore, for the comparison of factor 3, factor 4, and factor 5 , the average CCA value is below 0.7 , so that $\mathrm{KW}$ on factor 3 , factor 4 and factor 5 has a small number of $\mathrm{n}$ correlations. As shown in table 15, table 16 and table 17

Table 15. Comparison Factor 3 Result and CCA

\begin{tabular}{|c|c|c|c|c|c|}
\hline \multirow{2}{*}{ No } & \multirow{2}{*}{ KW } & \multirow{2}{*}{$\begin{array}{c}\text { Factor } 3 \\
\text { Result }\end{array}$} & \multicolumn{3}{|c|}{ CCA Result } \\
\hline & & & $n>0.7$ & $n>0.8$ & $n>0.9$ \\
\hline 1 & KW3 & 0.923 & 1 & 1 & 1 \\
\hline \multicolumn{6}{|c|}{ Table 16. Comparison Factor 4 Result and CCA } \\
\hline \multirow{2}{*}{ No } & \multirow{2}{*}{ KW } & \multirow{2}{*}{$\begin{array}{c}\text { Factor } 4 \\
\text { Result }\end{array}$} & \multicolumn{3}{|c|}{ CCA Result } \\
\hline & & & $n>0.7$ & $n>0.8$ & $\mathrm{n}>0.9$ \\
\hline 1 & KW10 & 0.957 & 2 & 2 & 2 \\
\hline
\end{tabular}

Table 17. Comparison Factor 5 Result and CCA

\begin{tabular}{|c|c|c|c|c|c|}
\hline \multirow{2}{*}{ No } & \multirow{2}{*}{ KW } & \multirow{2}{*}{$\begin{array}{c}\text { Factor 5 } \\
\text { Result }\end{array}$} & \multicolumn{3}{|c|}{ CCA Result } \\
\cline { 4 - 6 } & & & $\mathrm{n}>0.7$ & $\mathrm{n}>0.8$ & $\mathrm{n}>0.9$ \\
\hline 1 & KW42 & $\mathbf{0 . 9 5 3}$ & 2 & 2 & 2 \\
\hline
\end{tabular}

\subsubsection{Evaluation Data Audit}

Evaluation of audit data was carried out by selecting the instruments involved, including Kansei Word (Emotion) and Specimen. In the audit data, the emotions are collected and then given a checklist whether the emotions are OK or Not OK. If it is OK then it will be included in the analysis, if it is not OK then the KW will be given the statement "Exclude" or "Rephrase" and given a revision. For "Exclude" is given if the emotion is not an initial statement of the user's perception, then the emotion is replaced. While "Rephrase" is given if the emotion is the user's perception but is less representative to express the emotion so that the element of feeling and emotion that is close is sought. Table 18 is KW (Emotion) Audit Data

Tabel 18. KW (Emotion) Data Audit

\begin{tabular}{|c|l|l|l|}
\hline No & KE (Emotion) & $\begin{array}{r}\text { (Exclude I } \\
\text { Rephrase) }\end{array}$ & \multicolumn{1}{|c|}{ Revision } \\
\hline 1 & Sharp & - & - \\
\hline 2 & Formal & - & - \\
\hline$\ldots$ & $\ldots$ & $\ldots$ & $\ldots$ \\
\hline 32 & Uniform & Rephrase & Authentic \\
\hline 33 & Equidistant & Rephrase & Easy-to-Measure \\
\hline 34 & Equivalent & Exclude & Empty \\
\hline 35 & Digitasi & Rephrase & Sophisticated \\
\hline 36 & Interactive & Rephrase & Easy \\
\hline 37 & Representative & Rephrase & Vivid \\
\hline
\end{tabular}




\section{CONCLUSION}

From the results of the pilot analysis, it is concluded that the Factor Analysis resulted in the accumulation of Factor 1 (F1) and Factor 2 (F2) with a percentage of $74,679 \%$ with $\mathrm{F} 1$ worth $49.902 \%$ and $\mathrm{F} 2$ $24.777 \%$. Based on the comparison results of Factor 1 and CCA that has high significant are "Accurate", "Brilliant", "Sharp", "Wonderful", "Dynamic", "Beautiful", "Wide", "Well-Arranged", "Cool", "Authentic", "Elegant", "Formal", "Masculine". Emotion by comparison between Factor 2 and CCA that has high significant are "Easy-to-Measure" and "Bright". While the emotion of the comparison of Factor 3, Factor 4 and Factor 5 with CCA is still relatively low, because the number of correlations in CCA with other emotions is lower than 0.7.

\section{ACKNOWLEDGMENTS}

The Research Team would like to thank the Center for Research and Community Service (P3M) of the Sriwijaya State Polytechnic for research funding support. Also to the research partner team from Universiti Teknologi MARA (UiTM) Malaysia for their contribution to this research

\section{REFERENCES}

[1] Yulmaini, "Pengembangan Sistem Informasi
Geografis Penyebaran Klinik dan Pengguna Alat Kontrasepsi di Bandar Lampung," J. Inform., vol. 14, no. 1, pp. 36-49, 2014.

[2] S. W. Binabar, D. J. S. H. Siregar, and W. Pratama, "Geographic Information System for Mapping the Potency of Batik Industry Centre," J. Inf. Syst. Eng. Bus. Intell., vol. 5, no. 1, pp. 40-47, 2019.

[3] A. M. Lokman and M. Nagamachi, "Validation of Kansei Engineering Adoption in E-Commerce Web Design," Kansei Eng. Int. J., vol. 9, no. 1, pp. 21-27, 2009.

[4] E. N. M. Ibrahim, A. M. Lokman, and M. Nagamachi, "Exploring Kansei Attributes of the Emotional Design Preferences on Children ' S Wear in Malaysia."

[5] J. R. Chou, "A Kansei evaluation approach based on the technique of computing with words," $A d v$. Eng. Informatics, vol. 30, no. 1, pp. 1-15, 2016, doi: 10.1016/j.aei.2015.11.001.

[6] S. A. KADIR, A. M. LOKMAN, and T. TSUCHIYA, "Emotional Responses Towards Unity YouTube Videos: Experts vs. Viewers Perspectives," Int. J. Affect. Eng., 2021, doi: 10.5057/ijae.ijae-d-20-00033.

[7] J. Widiyanto, SPSS for Windows Untuk Analisis Data Statistik dan Penelitian. Surakarta: BP-FKIP UMS. Surakarta: BP-FKIP UMS, 2010 